\title{
Correction to: On fully intuitionistic fuzzy multiobjective transportation problems using different membership functions
}

\author{
Sumati Mahajan ${ }^{1} \cdot$ S. K. Gupta ${ }^{1}$ \\ ๑) Springer Science+Business Media, LLC, part of Springer Nature 2019
}

\section{Correction to: Annals of Operations Research https://doi.org/10.1007/s10479-019-03318-8}

This erratum is published because vendor overlooked corrections related to Theorems 3 to 5. In the proofs of the Theorems 3-5, please read " $<=$ " as " $\leq$ " and " $>=$ " as " $\geq$ ". In Page no. 17 (Theorem 5), line no. 7 from the bottom, read " $\delta^{*}>(>=) \hat{\delta}^{*}$ " as " $\delta^{*} \leq(<) \hat{\delta}$ ".

The original article has been thus corrected with the corrected references and thus updated.

Publisher's Note Springer Nature remains neutral with regard to jurisdictional claims in published maps and institutional affiliations.

The original article can be found online at https://doi.org/10.1007/s10479-019-03318-8.

Sumati Mahajan

sumatimahajan@pec.ac.in

S. K. Gupta

skgiitr@gmail.com

1 Department of Mathematics, Indian Institute of Technology Roorkee, Roorkee 247 667, India 